\title{
Economic and clinical outcomes of pegfilgrastim via prefilled syringe vs on-body injector: a real-world data analysis
}

\author{
Ali McBride, PharmD, MS, BCOP; Kim Campbell, PharmD, BCOP; Edward Li, PharmD, MPH; \\ Bridgette Schroader, PharmD, MPA, BCOP; David Campbell, PharmD, MS; and Weijia Wang, MS
}

\section{What is already known about this subject}

- Pegfilgrastim is available in a prefilled syringe (PFS) and on-body injector (OBI) dosage forms to decrease the incidence of infection in patients with nonmyeloid malignancies receiving myelosuppressive anticancer therapy.

- This analysis included data when only the originator product was available, and there are now 4 FDA-approved biosimilar pegfilgrastim PFS products that may generate substantial cost savings over time.

- A recent economic simulation model found that biosimilar pegfilgrastim PFS offers the greatest cost efficiency among all pegfilgrastim product options, including OBI.

\section{What this study adds}

- This is the first large real-world database analysis investigating the clinical and economic outcomes between PFS and OBI methods of pegfilgrastim administration.

- In a matched cohort of patients representing real-world utilization, there was no statistically or clinically meaningful difference in febrile neutropenia incidence between $\mathrm{OBI}$ and PFS methods of pegfilgrastim administration and no difference in total health care resource utilization or total costs.

\section{ABSTRACT}

BACKGROUND: Pegfilgrastim is available as a prefilled syringe (PFS) and an on-body injector (OBI). Whether the administration method of pegfilgrastim affects the effectiveness and health care resources has not been evaluated in the setting of routine care.

OBJECTIVE: To compare real-world clinical and economic outcomes between PFS and $\mathrm{OBI}$ methods of administration.

METHODS: This was a retrospective observational study in patients diagnosed with breast cancer or non-Hodgkin lymphoma who received myelosuppressive chemotherapy and prophylactic use of pegfilgrastim via PFS or OBI between January 1, 2017, and May 31, 2018, according to MarketScan research databases. A propensity score was used to match the PFS cohort 1:1 to the OBI cohort. Outcomes were compared among the matched cohorts using a generalized linear model and generalized estimating equations with log-link function.

RESULTS: 3,152 patients were identified. After matching, the final sample included 2,170 patients, representing 1,085 in each cohort. The incidence of febrile neutropenia (FN) in the first chemotherapy cycle was $1.01 \%$ for $\mathrm{OBI}(95 \% \mathrm{Cl}=0.56-1.82)$ vs $1.48 \%$ for PFS $(95 \%$

\section{Author affiliations}

Ali McBride, PharmD, MS, BCOP, Banner University Medical Center and University of Arizona Cancer Center, Tucson, AZ. Kim Campbell, PharmD, BCOP; Edward Li, PharmD, MPH; and Weijia Wang, MS, Sandoz, Inc, Princeton, NJ. Bridgette Schroader, PharmD, MPA, BCOP, and David Campbell, PharmD, MS, Xcenda, LLC, Tampa, FL.

\section{AUTHOR CORRESPONDENCE:}

Weijia Wang, 609.212.6191;

weijia.wang@sandoz.com

$J$ Manag Care Spec Pharm 2021;27(9):1230-38

Copyright $\odot 2021$, Academy of Managed Care Pharmacy. All rights reserved.

$\mathrm{Cl}=0.91-2.39 ; P=0.336)$. In all chemotherapy cycles (total cycles $=7,467$ ), the $\mathrm{FN}$ incidence was $0.91 \%$ for $\mathrm{OBI}(95 \% \mathrm{Cl}=0.64-1.30)$ vs $1.22 \%$ for PFS ( $95 \% \mathrm{Cl}=0.90-1.64 ; \mathrm{P}=0.214)$. There was no statistically significant difference in adjusted per-member per-month all-cause total cost health care resource utilization (HCRU) for hospitalizations, emergency department visits, and pharmacy claims.

CONCLUSIONS: In a matched cohort of patients representing real-world utilization, there was no statistically or clinically meaningful difference in $\mathrm{FN}$ incidence between $\mathrm{OBI}$ and PFS methods of pegfilgrastim 
administration. There was no difference in total HCRU or total costs. OBI and PFS methods of administration are both indicated for patients requiring prophylactic pegfilgrastim, which is important considering that biosimilar PFS options are now available.

Pegfilgrastim is a long-acting granulocyte colony-stimulating factor approved by the US Food and Drug Administration (FDA) to decrease the incidence of infection in patients with nonmyeloid malignancies receiving myelosuppressive anticancer therapy associated with a clinically significant incidence of febrile neutropenia (FN). ${ }^{1}$ It is available in 2 dosage forms: (1) a prefilled syringe (PFS) that is administered by health care providers or self-injected at least 24 hours after completion of chemotherapy, and (2) an on-body injector (OBI) that is affixed to the patient on the day of chemotherapy and auto-injects pegfilgrastim approximately 1 day later. ${ }^{1}$

The OBI was approved by the FDA in 2014 based on a phase 1 comparison of the pharmacokinetics and safety of pegfilgrastim administered by $\mathrm{OBI}$ and PFS. While the pharmacokinetics were comparable, the OBI cohort experienced numerically more adverse events. ${ }^{2}$ The OBI formulation has been linked to dose delivery failures, which have the potential to raise health care costs with FN-related hospitalizations. ${ }^{3}$

Recent economic simulation models found that biosimilar pegfilgrastim PFS offers the greatest cost efficiency among all pegfilgrastim product options, including OBI.,4 Considering the addition of 4 FDA-approved biosimilar pegfilgrastim PFS products, information about the comparability between OBI and PFS formulations is even more important, as the biosimilar products may generate substantial cost savings over time..$^{5-9}$ These cost models highlight the potential to lower overall costs and total cost of care through the avoidance of high-cost FN episodes..$^{10,11}$

With multiple product options, patients, providers, and payers require evidence that explicitly compares the outcomes among these 2 different drug delivery systems. The purpose of this study was to compare clinical and economic outcomes between PFS and OBI methods of pegfilgrastim administration.

\section{Methods}

\section{STUDY DESIGN AND DATA SOURCE}

This was a retrospective, observational, matched cohort study in patients diagnosed with breast cancer (BC) or
non-Hodgkin lymphoma (NHL) who received myelosuppressive chemotherapy and prophylactic use of pegfilgrastim via PFS or OBI. BC and NHL were chosen as proxy disease states representing populations with frequent use of primary FN prophylaxis. The data sources for this study were the MarketScan Commercial Claims and Encounters database and the MarketScan Medicare Supplemental and Coordination of Benefits database (ie, MarketScan research databases). These are deidentified national claims databases representing more than 150 large employer and health insurance plans containing more than 50 million participants. In addition to the Medicare population, this data set represents $>15 \%$ of the employer-sponsored, privately insured, and capitated US population aged less than 65 years. Medical data, pharmacy data, and enrollment information were collected.

\section{PATIENT AND COHORT IDENTIFICATION}

Adult commercial and Medicare health plan members who received their first cycle of chemotherapy for BC or NHL (defined as the index chemotherapy) with pegfilgrastim prophylaxis from January 1, 2017, to May 31, 2018 (index period), were included. This date range was chosen because pegfilgrastim was available through only 1 manufacturer during this time. Pegfilgrastim prophylaxis was defined as $\geq 1$ pharmacy or medical claim for pegfilgrastim, and the index date was defined as the first receipt of pegfilgrastim prophylaxis. Patients were required to be continuously enrolled at least 6 months before and after the index date and had to be chemotherapy-free for 5 years prior to the first chemotherapy administration identified during the index period. Patients were followed until the end of the index chemotherapy or up to a maximum of 6 cycles (Supplementary Figure 1, available in online article).

Evidence of $\mathrm{BC}$ or NHL was defined as $\geq 1$ diagnosis of cancer prior to the index date, either via inpatient or outpatient services; patients with both a BC and NHL diagnosis were excluded. Chemotherapy was defined as $\geq 1$ claim for a chemotherapeutic agent identified from medical outpatient services during the index period; patients with NHL must have received the following combination of agents as the initial chemotherapy regimen: $\mathrm{CHOP}$ (proxied by identification of cyclophosphamide, doxorubicin, and vincristine) with or without rituximab. Prednisone is part of this regimen but is not used for identification because of difficulty in selecting for dose. Patients with $\mathrm{BC}$ receiving myelosuppressive chemotherapy were further categorized based on the regimen's FN risk (high, intermediate, or other [Supplementary Table 1, available in online article]) based on National Comprehensive Cancer Network guidelines. ${ }^{12}$ 


\begin{tabular}{|c|c|}
\hline & $\begin{array}{c}\text { Unique } \\
\text { patients, } n\end{array}$ \\
\hline \multicolumn{2}{|l|}{ Chemotherapy } \\
\hline Medical outpatient 1/1/2017-5/31/2018 & 218,716 \\
\hline No chemotherapy in previous 5 years & 122,112 \\
\hline \multicolumn{2}{|l|}{ Pegfilgrastim } \\
\hline Pegfilgrastim 1/1/2017-5/31/2018 (index date) & 26,283 \\
\hline $\mathrm{OBI}$ & 8,645 \\
\hline PFS & 18,202 \\
\hline Missing & 7,074 \\
\hline \multicolumn{2}{|l|}{ Join } \\
\hline Chemotherapy and pegfilgrastim & \multirow{2}{*}{12,880} \\
\hline Pegfilgrastim and chemotherapy $\leq 5$ days apart & \\
\hline \multicolumn{2}{|l|}{ Enrollment } \\
\hline 6 months before index date & 9,854 \\
\hline 6 months after index date & 8,001 \\
\hline \multicolumn{2}{|l|}{ Adult } \\
\hline Adult on index year & 7,968 \\
\hline \multicolumn{2}{|l|}{ Cancer } \\
\hline$\geq 1$ diagnosis of cancer before index date & 5,667 \\
\hline \multicolumn{2}{|l|}{ Exclusion } \\
\hline Myeloid cancer & 5,642 \\
\hline Pregnancy & 5,539 \\
\hline Clinical trial participation & 5,427 \\
\hline HIV positive & 5,408 \\
\hline Hematopoietic stem cell transplantation & 5,385 \\
\hline Other types of nonmyeloid cancers & 4,849 \\
\hline Missing region & 4,840 \\
\hline Switched between OBI and PFS & 3,152 \\
\hline Final total & 3,152 \\
\hline Matched cohort & 2,170 \\
\hline $\mathrm{OBI}=$ on-body injector; $\mathrm{PFS}=$ prefilled syringe. & \\
\hline
\end{tabular}

Patients were excluded if they had evidence of other cancer, were pregnant, were participating in a clinical trial, were HIV-positive, had prior history of hematopoietic stem cell transplant, and/or had missing values of baseline and clinical characteristics.

Current Procedural Terminology (CPT) codes were used to assign patients to the PFS cohort (CPT: 96372) or OBI cohort (CPT: 96377). Patients who switched between PFS and OBI were excluded, so patients within each cohort were composed of mutually exclusive PFS or OBI methods of administration across all chemotherapy cycles.

\section{OUTCOME MEASURES}

The clinical outcomes included FN incidence among the first chemotherapy cycle and across all cycles. FN was defined as having a claim with a diagnosis code for neutropenia plus fever or infection on the same date, captured in each chemotherapy cycle from both medical inpatient and outpatient services, as previously validated..$^{13} \mathrm{FN}$ incidence across all cycles was calculated based on the number of FN events across all chemotherapy cycles.

Economic outcomes included all-cause health care resource utilization (HCRU) and all-cause costs from a payer perspective in the 6-month follow-up period. Costs and HCRU were reported for pharmacy and medical categories, including inpatient hospitalization, outpatient visits (emergency department [ED] visits, outpatient office visits), and other visits. Length of stay was captured among those with at least 1 hospitalization. Costs were adjusted using the annual medical component of the Consumer Price Index to 2020 US dollars and both all-cause HCRU and costs were reported per patient per month (PPPM).

\section{STATISTICAL ANALYSIS}

Descriptive statistics were provided for all study variables. To reduce potential selection bias, a propensity score model was developed using logistic regression to estimate the probability of patients receiving OBI or PFS methods of administration. Covariates that were adjusted in the logistic regression included sex, cancer type, geographic region, insurance type (commercial vs Medicare), chemotherapy FN risk category (high, intermediate, other), Quan-Charlson comorbidity score, and patient-specific FN risk factors (metastatic bone disease, baseline radiation, baseline surgery, baseline liver and renal dysfunction, history of persistent neutropenia, and age $>65$ years). Based on the propensity score, patients using PFS were 1:1 matched to patients using OBI using the greedy nearest neighbor matching algorithm. ${ }^{14}$

To compare the FN incidence among the first chemotherapy cycle, a logistic regression model was developed among the matched cohorts. To compare the FN incidence among all chemotherapy cycles, a generalized estimating equation (GEE) model with binomial distribution, log-link function, and exchangeable correlation structure was developed to account for the fact that the probability of experiencing an FN event during 1 chemotherapy cycle was dependent on previous chemotherapy cycles (hence, the assumption of independent samples was violated). This model estimated the average FN incidence across all chemotherapy cycles 
Economic and clinical outcomes of pegfilgrastim via prefilled syringe vs on-body injector: a real-world data analysis 1233

\section{TABLE 2 Baseline Characteristics ${ }^{\mathrm{a}}$}

\begin{tabular}{|c|c|c|c|c|c|c|c|}
\hline \multirow[b]{2}{*}{ Variable } & \multicolumn{3}{|c|}{ Unadjusted cohort } & \multicolumn{3}{|c|}{ Adjusted cohort } & \multirow{2}{*}{$\begin{array}{l}\text { Standardized } \\
\text { difference }^{\mathrm{b}}, \%\end{array}$} \\
\hline & All $(n=3,152)$ & OBI $(n=1,088)$ & PFS $(n=2,064)$ & All $(n=2,170)$ & OBI $(n=1,085)$ & PFS $(n=1,085)$ & \\
\hline Age, median (range) & $54(23-88)$ & $53(24-84)$ & $54(23-88)$ & $53(46-59)$ & $53(46-60)$ & $53(46-59)$ & NA \\
\hline Sex, female (\%) & 3,018 (95.75) & $1,053(96.78)$ & $1,965(95.20)$ & 2,105 (97.00) & $1,050(96.77)$ & 1,055 (97.24) & -2.35 \\
\hline \multicolumn{8}{|l|}{ US region, n (\%) } \\
\hline North Central & $640(20.30)$ & $234(21.51)$ & 406 (19.67) & $460(21.20)$ & $233(21.47)$ & $227(20.92)$ & \multirow{4}{*}{ NA } \\
\hline Northeast & $462(14.66)$ & $153(14.06)$ & 309 (14.97) & 309 (14.24) & $153(14.10)$ & $156(14.38)$ & \\
\hline South & $1,629(51.68)$ & $599(55.06)$ & $1,030(49.90)$ & $1,197(55.16)$ & $597(55.02)$ & $600(55.30)$ & \\
\hline West & $421(13.36)$ & $102 \quad(9.38)$ & $319(15.46)$ & $204 \quad(9.40)$ & $102 \quad(9.40)$ & $102 \quad(9.40)$ & \\
\hline \multicolumn{8}{|l|}{ Insurance type, n (\%) } \\
\hline Commercial & $2,888 \quad(91.62)$ & $1,054(96.88)$ & $1,834(88.86)$ & $2,100(96.77)$ & 1,051 (96.87) & 1,049 (96.68) & \multirow{2}{*}{0.73} \\
\hline Medicare & $264 \quad(8.38)$ & $34 \quad(3.13)$ & $230(11.14)$ & $70 \quad(3.23)$ & $34 \quad(3.13)$ & $36 \quad(3.32)$ & \\
\hline \multicolumn{8}{|l|}{ Diagnosis, n (\%) } \\
\hline$B C$ & 2,935 (93.12) & $1,032(94.85)$ & $1,903(92.20)$ & $2,053(94.61)$ & $1,031(95.02)$ & 1,022 (94.19) & \multirow{2}{*}{-1.51} \\
\hline $\mathrm{NHL}$ & $217 \quad(6.88)$ & $56 \quad(5.15)$ & $161 \quad(7.80)$ & $117 \quad(5.39)$ & $54 \quad(4.98)$ & $63 \quad(5.81)$ & \\
\hline \multicolumn{8}{|l|}{ Regimen FN risk, n (\%) } \\
\hline High & $2,427(77.00)$ & 831 (76.38) & $1,596(77.33)$ & $1,669(76.91)$ & 831 (76.59) & $838(77.24)$ & \multirow{3}{*}{ NA } \\
\hline Intermediate & $692(21.95)$ & $247(22.70)$ & $445(21.56)$ & $476(21.94)$ & $244(22.49)$ & $232(21.38)$ & \\
\hline Other & $33 \quad(1.05)$ & $10 \quad(0.92)$ & $23 \quad(1.11)$ & $25 \quad(1.15)$ & $10 \quad(0.92)$ & $15 \quad(1.38)$ & \\
\hline \multicolumn{8}{|c|}{ Quan-Charlson comorbidity score, n (\%) } \\
\hline $1-2$ & $1,139(36.14)$ & 397 (36.49) & $742(35.95)$ & $784(36.13)$ & $396(36.50)$ & $388(35.76)$ & \multirow{3}{*}{ NA } \\
\hline $3-4$ & $504(15.99)$ & $165(15.17)$ & $339(16.42)$ & $326(15.02)$ & $163(15.02)$ & $163(15.02)$ & \\
\hline $5+$ & 1,509 (47.87) & $526(48.35)$ & 983 (47.63) & $1,060(48.85)$ & $526(48.48)$ & 534 (49.22) & \\
\hline
\end{tabular}

Day of pegfilgrastim claim in reference to chemotherapy, $\mathrm{n}(\%)$

\begin{tabular}{|c|c|c|c|c|c|c|c|c|}
\hline D0 & 1,294 & $(41.5)$ & NA & $1,294 \quad(62.69)$ & NA & NA & NA & \multirow{3}{*}{ NA } \\
\hline $\mathrm{D} 1$ & 1,748 & $(55.5)$ & $1,070(98.35)$ & $678(32.85)$ & NA & NA & NA & \\
\hline $\mathrm{D} 2+$ & 1,074 & $(34.1)$ & $982 \quad(1.65)$ & $92 \quad(4.46)$ & NA & NA & NA & \\
\hline
\end{tabular}

FN risk factors, $\mathrm{n}(\%)$

\begin{tabular}{|c|c|c|c|c|c|c|c|}
\hline Bone metastases & NR & NR & NR & NR & NR & NR & 0 \\
\hline Baseline radiation & $105 \quad(3.33)$ & $24 \quad(2.21)$ & $81 \quad(3.92)$ & $44 \quad(2.03)$ & $24 \quad(2.21)$ & $20 \quad(1.84)$ & -2.14 \\
\hline Recent surgery & $2,643(83.85)$ & $898(82.54)$ & $1,745(84.54)$ & 1,790 (82.49) & $896(82.58)$ & $894(82.40)$ & -0.50 \\
\hline Baseline liver dysfunction & $241 \quad(7.65)$ & $73 \quad(6.71)$ & $168 \quad(8.14)$ & $141 \quad(6.50)$ & $73 \quad(6.73)$ & $68 \quad(6.27)$ & -1.76 \\
\hline Baseline renal dysfunction & $87 \quad(2.76)$ & $23 \quad(2.11)$ & $64 \quad(3.10)$ & $47 \quad(2.17)$ & $23 \quad(2.12)$ & $24 \quad(2.21)$ & 0.58 \\
\hline $\begin{array}{l}\text { History of persistent } \\
\text { neutropenia }\end{array}$ & $24 \quad(0.76)$ & NR & $18 \quad(0.87)$ & $11 \quad(0.51)$ & NR & NR & -1.10 \\
\hline Age $\geq 65$ years & $303 \quad(9.61)$ & $54 \quad(4.96)$ & $249(12.06)$ & 105 (4.84) & $51 \quad(4.70)$ & $54 \quad(4.98)$ & 1.0 \\
\hline
\end{tabular}

a Results with $\leq 6$ patients were not reported to maintain anonymity.

${ }^{b}$ Standardized differences were estimated only for binary categorical variables.

$B C=$ breast cancer; $D=$ day; $F N=$ febrile neutropenia; $N A=$ not applicable; $N H L=$ non-Hodgkin lymphoma; $N R=$ not reported; $O B I=$ on-body injector; $P F S=$ prefilled syringe. 


\begin{tabular}{|c|c|c|c|c|c|}
\hline \multirow[b]{2}{*}{ Population } & \multicolumn{2}{|c|}{ OBI } & \multicolumn{2}{|c|}{ PFS } & \multirow[b]{2}{*}{$P$ value } \\
\hline & FN incidence, \% & $95 \% \mathrm{Cl}$ & FN incidence, $\%$ & $95 \% \mathrm{Cl}$ & \\
\hline \multicolumn{6}{|l|}{ First cycle } \\
\hline Unadjusted $(n=3,152)$ & 1.01 & $0.42-1.61$ & 1.55 & $1.02-2.08$ & 0.215 \\
\hline Adjusteda $(n=2,170)$ & 1.01 & $0.56-1.82$ & 1.48 & $0.91-2.39$ & 0.336 \\
\hline \multicolumn{6}{|l|}{ All cycles } \\
\hline Unadjusted $($ cycles $=11,196)$ & 0.81 & $0.56-1.18$ & 1.00 & $0.79-1.26$ & 0.879 \\
\hline Adjusteda $($ cycles $=7,467)$ & 0.91 & $0.64-1.30$ & 1.22 & $0.90-1.64$ & 0.214 \\
\hline
\end{tabular}

${ }^{a}$ Adjusted for sex, cancer type, region, insurance type, regimen FN risk level, Quan-Charlson comorbidity score, and FN risk factors.

$\mathrm{FN}=$ febrile neutropenia; $\mathrm{OBI}=$ on-body injector; $\mathrm{PFS}=$ prefilled syringe.

and controlled for the same covariates as the propensity score model (except for the removal of metastatic bone disease from the final model due to nonconvergence of the GEE model estimation).

All-cause HCRU and costs were compared in the matched cohort by using multivariable analyses. Adjusted PPPM costs (ie, medical and pharmacy costs) were estimated using generalized linear models (GLMs) with Tweedie distribution (compound Poisson-gamma distribution) and log-link function to account for differences in baseline characteristics. Adjusted PPPM HCRU (ie, hospitalizations, lengths of stay, ED visits, office visits, and pharmacy claims) was estimated using GLM with Poisson distribution and log-link function. Adjusted least-square means and 95\% CIs of the predicted values were reported. All statistical analyses were performed with SAS version 9.4 (SAS Institute).

\section{Results}

\section{PATIENT CHARACTERISTICS}

A total of 3,152 patients were identified, including 1,088 who received pegfilgrastim prophylaxis via OBI and 2,064 who received it via PFS (Table 1). After propensity score matching (adjusted cohort), the final sample included 2,170 matched patients, representing 1,085 in each administration cohort. The median age was 53 years (range: 23-87), and the majority of patients were female (96.9\%). BC was more common than NHL (94.6\% vs 5.3\%, respectively), and most regimens were high risk for FN (77\%). Most of the patients had 1 FN risk factor (75.7\%), and $82.8 \%$ of patients had recent surgery in the baseline period. Baseline characteristics for the unadjusted and adjusted samples are shown in Table 2.

\section{FN INCIDENCE}

The rates of FN within each pegfilgrastim cohort were low (Table 3). During the first cycle of chemotherapy, there was no statistically significant difference in FN incidence between the OBI or PFS cohorts $(1.01 \%$ [95\% CI=0.56-1.82] vs $1.48 \%$ [95\% $\mathrm{CI}=0.91-2.39]$, respectively; $\mathrm{P}=0.336)$. When considering all chemotherapy cycles (total cycles $=7,467$ ), there was also no difference in FN incidence between the OBI or PFS cohorts $(0.91 \%$ [ $95 \% \mathrm{CI}=0.64-1.30]$ vs $1.22 \%$ [95\% $\mathrm{CI}=0.90-1.64]$, respectively; $\mathrm{P}=0.214)$. Similarly, there was no statistically significant difference in the rates of FN in the unadjusted cohort.

\section{ADJUSTED ALL-CAUSE TOTAL COST}

There was no statistically significant difference in total allcause adjusted cost between OBI and PFS cohorts $(\$ 21,745$ $[95 \% \mathrm{CI}=\$ 20,944-\$ 22,578]$ vs $\$ 20,655 \quad[95 \% \mathrm{CI}=\$ 19,900-$ $\$ 21,438]$ PPPM, respectively; $\mathrm{P}=0.055$; Table 4). There were also no differences in medical, ED, and inpatient hospitalization costs. The OBI cohort was associated with higher outpatient costs $(\$ 14,737$ [95\% CI, $\$ 13,812-\$ 15,724]$ vs $\$ 10,961$ [95\% CI $=\$ 10,247-\$ 11,725]$ PPPM; P $<0.001)$, while the PFS cohort was associated with higher pharmacy costs $(\$ 319$ $[95 \% \mathrm{CI}=\$ 290-\$ 350]$ vs $\$ 226[95 \% \mathrm{CI}=\$ 205-\$ 248] \mathrm{PPPM}$; $\mathrm{P}<0.001)$.

\section{ADJUSTED ALL-CAUSE TOTAL HCRU}

There were no statistically significant differences in PPPM number of hospitalizations, ED visits, or pharmacy claims (Table 5). Among 409 patients with $\geq 1$ hospitalization, patients in the PFS cohort had significantly longer stays than the OBI cohort (4.71 days [95\% $\mathrm{CI}=4.44-4.99]$ vs 3.81 days $[95 \% \mathrm{CI}=3.55-4.09] \mathrm{PPPM}$; $\mathrm{P}<0.001$ ). Patients who received pegfilgrastim via $O B I$ had a higher mean number of office visits than those who received PFS (3.09 visits 


\begin{tabular}{|c|c|c|c|c|}
\hline $\begin{array}{c}\text { Cost } \\
\text { component, PPPM }\end{array}$ & $\begin{array}{c}\text { Method of } \\
\text { administration }\end{array}$ & Cost (mean), \$ & $95 \% \mathrm{Cl}, \mathbf{\$}$ & $P$ value \\
\hline \multirow{2}{*}{ Total } & $\mathrm{OBI}$ & 21,745 & $20,944-22,578$ & \multirow{2}{*}{0.055} \\
\hline & PFS & 20,655 & $19,900-21,438$ & \\
\hline \multirow{2}{*}{ Medical } & $\mathrm{OBI}$ & 21,498 & $20,096-22,331$ & \multirow{2}{*}{0.374} \\
\hline & PFS & 20,321 & $19,570-22,000$ & \\
\hline \multirow{2}{*}{ Outpatient } & $\mathrm{OBI}$ & 14,737 & $13,812-15,724$ & \multirow{2}{*}{$<0.001$} \\
\hline & PFS & 10,961 & $10,247-11,725$ & \\
\hline \multirow{2}{*}{ Emergency } & $\mathrm{OBI}$ & 122 & $103-145$ & \multirow{2}{*}{0.899} \\
\hline & PFS & 124 & $104-148$ & \\
\hline \multirow{2}{*}{ Inpatient } & $\mathrm{OBI}$ & 1,236 & $1,039-1,471$ & \multirow{2}{*}{0.777} \\
\hline & PFS & 1,290 & $1,085-1,534$ & \\
\hline \multirow{2}{*}{ Other } & $\mathrm{OBI}$ & 5,371 & $4,975-5,799$ & \multirow{2}{*}{$<0.001$} \\
\hline & PFS & 7,912 & $7,342-8,526$ & \\
\hline \multirow{2}{*}{ Pharmacy } & $\mathrm{OBI}$ & 226 & $205-248$ & \multirow{2}{*}{$<0.001$} \\
\hline & PFS & 319 & $290-350$ & \\
\hline
\end{tabular}

${ }^{a}$ Costs are reported in 2020 US dollars.

$\mathrm{OBI}=$ on-body injector; $P F \mathrm{~S}=$ prefilled syringe; $P P P M=$ per patient per month.

[95\% CI $=2.98-3.19]$ vs 2.61 visits $[95 \%$ $\mathrm{CI}=2.52-2.71]$ PPPM; $\mathrm{P}<0.001$ ).

\section{Discussion}

This study showed that there is no difference in FN incidence in both the first cycle and over all cycles of chemotherapy when administering pegfilgrastim prophylaxis via PFS or OBI. Additionally, there is no difference in all-cause total cost and HCRU.

To our knowledge, this is the first published large real-world database study investigating clinical and economic outcomes with 2 methods of pegfilgrastim administration. Previous studies have been conducted that evaluated the clinical outcomes based on the method of administration. In a retrospective single-center study, Townley et al compared the incidence of grade 4 neutropenia in patients receiving pegfilgrastim, regardless of cancer type, as PFS vs OBI in a matched cohort. A total of
58 patients were included in each cohort, and there was no difference in the incidence of grade 4 neutropenia ( 3 patients in the OBI cohort vs 1 patient in the PFS cohort; $\mathrm{P}=0.618$ ). They reported 4 patients with device failures in the OBI cohort; no cost data were reported..$^{15}$ Jindal et al reported outcomes in 120 patients with any cancer type in a single center (60 receiving PFS, 60 receiving OBI) and concluded that there was no difference in FN incidence (16.7\% PFS vs 8.3\% OBI; $\mathrm{P}=0.17$ ). Three OBI device failures were reported. ${ }^{16}$

One abstract recently reported an analysis of the MarketScan database using a similar definition of $\mathrm{FN}$ as our study. They identified that among 10,854 eligible patients, the incidence of FN was significantly lower in the OBI cohort vs the PFS cohort over all chemotherapy cycles $(1.3 \%$ vs $1.7 \%$; $\mathrm{P}=0.01) .{ }^{17}$ However, the study authors did not control for bias related to confounding by indication and statistical overpowering, which prevents any inference of a causal relationship between administration method and outcome from being made.

Our study utilized stringent inclusion and exclusion criteria to develop 2 cohorts with equivalent baseline characteristics for appropriate comparison and a detailed statistical plan. As part of this, a propensity score match was utilized as the most logical approach to balance the number of patients in the OBI and PFS cohorts and account for time-to-event analysis. ${ }^{18}$ We also applied a GLM including GEEs to provide estimates that fit to the distribution of the outcomes variables without making gross assumptions, which might deviate our findings from true effects. Finally, with more than 2,100 patients within our matched sample size, our study had an estimated $87.2 \%$ power to detect a clinically significant difference of $0.83 \%$ in FN incidence. These add to the strength of our conclusions.

In addition to clinical equivalence, our study illustrated that there are no differences in all-cause total cost or all-cause HCRU between OBI and PFS administration during an era where only 1 pegfilgrastim product was available. This is an especially important consideration, as 4 biosimilars to pegfilgrastim have been approved by the FDA, but all are only available in the PFS dosage form. An economic simulation had previously shown the potential advantage of assured FN prophylaxis with biosimilar PFS administration over both reference product PFS and OBI administration, owing to the lower acquisition cost of biosimilar pegfilgrastim. ${ }^{3}$ Our realworld analysis confirms that there is no difference in cost or HCRU associated with the method of pegfilgrastim administration, thus supporting the potential real-world cost efficiency of biosimilar pegfilgrastim due to its overall lower acquisition cost. 


\begin{tabular}{|c|c|c|c|c|}
\hline $\begin{array}{c}\text { Cost } \\
\text { component, PPPM }\end{array}$ & $\begin{array}{c}\text { Method of } \\
\text { administration }\end{array}$ & Mean & $95 \% \mathrm{Cl}$ & $P$ value \\
\hline \multirow{2}{*}{ Hospitalizations } & $\mathrm{OBI}$ & 0.08 & $0.07-0.10$ & \multirow{2}{*}{0.674} \\
\hline & PFS & 0.09 & $0.07-0.11$ & \\
\hline \multirow{2}{*}{ LOS $^{a}$, days } & $\mathrm{OBI}$ & 3.81 & $3.55-4.09$ & \multirow{2}{*}{$<0.001$} \\
\hline & PFS & 4.71 & 4.44-4.99 & \\
\hline \multirow{2}{*}{ ED visits } & $\mathrm{OBI}$ & 0.07 & 0.05-0.09 & \multirow{2}{*}{0.200} \\
\hline & PFS & 0.08 & $0.07-0.10$ & \\
\hline \multirow{2}{*}{ Office visits } & $\mathrm{OBI}$ & 3.09 & $2.98-3.19$ & \multirow{2}{*}{$<0.001$} \\
\hline & PFS & 2.61 & $2.52-2.71$ & \\
\hline \multirow{2}{*}{ Pharmacy claims } & $\mathrm{OBI}$ & 2.12 & $2.04-2.21$ & \multirow{2}{*}{0.685} \\
\hline & PFS & 2.10 & $2.01-2.18$ & \\
\hline
\end{tabular}

a Measured among those with $\geq 1$ hospitalization $(n=409)$.

$E D=$ emergency department; $L O S=$ length of stay; $O B I=$ on-body injector; $P F S=$ prefilled syringe $; P P P M=p e r$ patient per month.

When considering specific components of total cost and HCRU, we did observe differences. First, patients who received OBI administration had more outpatient visits than patients receiving PFS. This may be due to OBI device failures necessitating return visits for administration of pegfilgrastim or filgrastim. Previous studies have reported OBI failure rates ranging from $1.7 \%$ to $6.9 \%{ }^{15,19,20}$ A recent model simulation found that the incremental cycle 1 cost for a hospitalization in patients with NHL who had OBI failure rates of $2 \%$ to $7 \%$ ranged from $\$ 600,569$ to $\$ 3,562,697 .{ }^{21}$ Additionally, patients who received OBI had higher outpatient costs, while those who received PFS had higher pharmacy costs. One possible explanation for these cost differences may be the dynamics of formulary design and distribution channels. For example, patients receiving OBI would likely receive placement of the device in a doctor's office, reflected in outpatient costs, while those receiving PFS may have a wider diversity of distribution methods such as via specialty pharmacies or other white or brown bagging methods that would then be reflected in pharmacy costs.

When multiple methods of administration are available and seemingly equivalent in outcomes and cost, it is important to consider patient and provider preference. Hauber et al reported preferences in 200 physicians and 200 patients. Overall, patients preferred the method of administration with which they had experience. Clinician preferences varied based on whether the patient was clinically compromised. The main burden associated with PFS was returning for clinic visits. ${ }^{22}$ In our study, there were no differences in FN incidence or cost in patients who received pegfilgrastim on day 0 (PFS) or day 1 (PFS or OBI; OBI receipt was considered to be day 1 as the administration of pegfilgrastim occurs approximately 1 day after placement). Interestingly, we did find that patients who received pegfilgrastim via OBI had a higher mean number of office visits than those who received PFS. Saif et al reported acceptance of OBI in a racially diverse population. They found $22 \%$ of OBI orders were refused for reasons including dislike of a bulky attachment, request for kit placement on the stomach instead of the arm, fear of unwitnessed drug administration, fear of reaction, disposal at home, fear of pain, and lack of confirmation of dose administration. ${ }^{23}$ The clinical equivalence and cost equivalence of OBI and PFS in this study support the use of either method of administration and allow for patient and physician preference to be accounted for during real-world practice.

\section{LIMITATIONS}

This study is limited by the biases inherent in retrospective claims studies and the difficulty in accurately identifying patients experiencing FN. The database may have misclassifications or missing data points. For example, approximately half of our patients had a date of billing of pegfilgrastim on the same day of chemotherapy. Misclassification between billing and administration dates may occur, warranting the need for clinical trials to compare the efficacy of sameday use vs later use of pegfilgrastim among patients with cancer undergoing chemotherapy. Further, OBI failures cannot be identified through claims data; we can only infer a potential failure based on clinic visits after chemotherapy and OBI claims.

We utilized rigorous review of chemotherapy agents and a matched cohort to achieve a homogenous population, minimizing selection bias and the effect of missing data. Additionally, by including chemotherapy treatments in the model, further confounding effects could be removed. However, while the selection of a homogenous population increased the internal validity of our study, it is also a limitation. Because we only included NHL and BC populations, our results may not be applicable to other cancer types. The majority (>95\%) of the patients in this analysis were 
female, which also limits the external validity of our results.

Next, this analysis only included reference pegfilgrastim. Given the recent approvals of pegfilgrastim biosimilars, it will take time for these products to gain market share to conduct a meaningful comparative study. This lack of inclusion reduces the generalizability of our results, but we would expect HCRU costs to be lower over time with the introduction of biosimilars.

The ability to infer causal effects from retrospective analyses is lower than that of a randomized prospective trial. Every effort was made to increase the internal validity of this study, but unmeasurable or unobserved variables and confounders that influence treatment assignment may still be missing from the models.

\section{Conclusions}

OBI pegfilgrastim administration has been touted as a more convenient method of administration that decreases a patient's need for additional clinic visits. However, our work shows that there is no difference in FN incidence, total HCRU, or total costs, indicating no difference between PFS and OBI method of administration. Further, the potential for device failure with OBI administration in the era of COVID-19 suggests a potential advantage for the use of PFS administration by patients in their homes for guaranteed prophylaxis and decreased health care exposure. Finally, the introduction of biosimilar pegfilgrastim PFS to the market may allow for a clinically equivalent and more cost-efficient option for FN prophylaxis.

\section{DISCLOSURES}

This study was funded by Sandoz, Inc. Wang, Li, and K. Campbell are employees of Sandoz, Inc. Schroader and D. Campbell are employees of Xcenda, which was contracted by Sandoz, Inc., to provide study and manuscript development. McBride reports receiving payment from Sandoz, Inc., as a consultant, unrelated to this study; Coherus for advisory board and speaker engagements; and Pfizer for advisory board participation during the time of this study.

\section{ACKNOWLEDGMENTS}

The authors thank Al Zabiby Anas and Saipoojitha Gudiboina for their support in programming and data analyses.

\section{REFERENCES}

1. Neulasta. Prescribing information. Amgen; 2020. Accessed April 26, 2021. https://www.pi.amgen.com/ /media/ amgen/repositorysites/pi-amgen-com/ neulasta/neulasta_pi_hcp_english.pdf

2. Yang BB, Morrow PK, Wu X, Moxness M, Padhi D. Comparison of pharmacokinetics and safety of pegfilgrastim administered by two delivery methods: on-body injector and manual injection with a prefilled syringe. Cancer Chemother Pharmacol. 2015;75(6):1199-206.

3. McBride A, Krendyukov A, Mathieson N, et al. Febrile neutropenia hospitalization due to pegfilgrastim on-body injector failure compared to single-injection pegfilgrastim and daily injections with reference and biosimilar filgrastim: US cost simulation for lung cancer and non-Hodgkin lymphoma. J Med Econ. 2020;23(1):28-36.

4. Morgane C, Mouslim DVM, Trujillo AJ, Alexander GC, Segal JB. Association between filgrastim biosimilar availability and changes in claim payments and patient out-of-pocket costs for biologic filgrastim products. Value Health. 2020;23(12):1599-605.

5. Mulcahy AW, Hlavka JP, Case SR. Biosimilar cost savings in the United States: initial experience and future potential. Rand Health Q. 2018;7(4):3.
6. Fulphila. Prescribing information. Mylan GmbH; 2018. Accessed April 26, 2021. https://www.accessdata.fda.gov/drugsatfda_docs/ label/2018/761075s000lbl.pdf

7. Nyvepria. Prescribing information. Pfizer, Inc.; 2020. Accessed April 26, 2021. https://www.accessdata.fda.gov/drugsatfda docs/label/2020/761111lbl.pdf

8. Udenyca. Prescribing information. Coherus BioSciences, Inc.; 2018. Accessed April 26, 2021. https://www. accessdata.fda.gov/drugsatfda_docs/ label/2018/761039s000lbl.pdf

9. Ziextenzo. Prescribing information. Sandoz; 2019. Accessed April 26, 2021. https://www.accessdata.fda.gov/drugsatfda docs/label/2019/761045lbl.pdf

10. Wang JX, Lopez SE, Chan A. Economic burden of chemotherapy-induced febrile neutropenia in patients with lymphoma: a systematic review. Crit Rev Oncol Hematol. 2015;94(2):201-12.

11. Baumgardner J, Shahabi A, Zacker C, Lakdawalla D. Cost variation and savings opportunities in the Oncology Care Model. Am J Manag Care. 2018;24(12):618-23.

12. National Comprehensive Cancer Network (NCCN) Clinical Practice Guidelines in Oncology. Hematopoietic growth factors version 2.2020. Updated January 27, 2020. Accessed December 14, 2020. https://www.nccn.org/professionals/physician gls/pdf/growthfactors.pdf

13. Weycker D, Sofrygin O, Seefeld K, Deeter RG, Legg J, Edelsberg J. Technical evaluation of methods for identifying chemotherapy-induced febrile neutropenia in healthcare claims databases. BMC Health Serv Res. 2013;13:60.

14. Austin PC. A comparison of 12 algorithms for matching on the propensity score. Stat Med. 2014;33:1057-69.

15. Townley C, Porter C, McMullen N. Comparing grade 4 neutropenia associated with pegfilgrastim administered via the onpro device versus manual injection with a prefilled syringe. J Hematol Oncol Pharm. 2018;8(3):119-25. 
16. Jindal A, Kover J, Raduka V, O'Brien TE. Incidence of neutropenic fever at a safety net hospital in cancer chemotherapy patients receiving prophylactic pegfilgrastim manual injection compared to the on-body auto-injector. Blood. 2018;132(suppl 1):4709.

17. Shah N, Hatfield M, Lawrence T, Manjelievskaia J, Moynihan M, Bonafede $\mathrm{M}$. Incidence of febrile neutropenia in chemotherapy cycles with pegfilgrastim receipt via on-body injector (Onpro) versus pre-filled syringe. J Manag Care Spec Pharm. 2020;26(4-a):S1-S96. doi: 10.18553/ jmcp.2020.26.4-a.s1

18. Austin PC, Fine JP. Propensity-score matching with competing risks in survival analysis. Stat Med. 2019;38(5):751-77.
19. Joshi RS, Egbuna OI, Cairns AS, et al. Performance of the pegfilgrastim on-body injector as studied with placebo buffer in healthy volunteers. Curr Med Res Opin. 2017;33(2):379-84.

20. Stuessy P, Sanchez FA, Schober M. Retrospective review of pegfilgrastim on-body injector delivery rates in a large health system [abstract e18273]. J Clin Oncol. 2017;35(15 suppl):e18273.

21. McBride A, Krendyukov A, Mathieson N, et al. Febrile neutropenia hospitalization due to pegfilgrastim on-body injector failure compared to single-injection pegfilgrastim and daily injections with reference and biosimilar filgrastim: US cost simulation for lung cancer and non-Hodgkin lymphoma. J Med Econ. 23;1:28-36.
22. Hauber AB, Mange B, Price MA, et al. Administration options for pegfilgrastim prophylaxis: patient and physician preferences from a cross-sectional survey. Support Care Cancer. 2018;26:251-60.

23. Saif MW, Hackenyos DW, Smith MH, Healey P, Relias V, Wasif K. Racial differences in accepting pegfilgrastim onpro kit (on-body injector) use among cancer patients. Clin Oncol (Las Vegas). 2019;1(6):1026. 